\title{
FORUM
}

\section{Ende gut - alles gut? Anmerkungen zum Reformvertrag}

\author{
Klaus Hänsch*
}

In meinem parlamentarischen Leben habe ich viele EU-Gipfel politisch begleitet, an einigen auch teilgenommen. Ein Europäischer Rat, der in Vorbereitung und Ergebnis dem vom 21./22. Juni 2007 vergleichbar wäre, ist nicht darunter. Noch nie ist eine Regierungskonferenz mit einem so präzisen und schlüssigen Mandat für eine Reform der Verträge auf den Weg gebracht worden. Noch nie allerdings konnte ein Gipfel auf einer so kohärenten Grundlage entscheiden, wie es der vom Europäischen Konvent entworfene und von den Staatsund Regierungschefs im Oktober 2004 beschlossene ,Vertrag über eine Verfassung für Europa“ war. Überdies war die politische Konstellation nach dem Regierungswechsel in Frankreich und nachdem 25 Regierungen ihren Willen bekundet hatten, einen neuen Anlauf noch vor der Europawahl 2009 zu wagen, außergewöhnlich günstig.

\section{Änderungsvertrag: Substanz, Vertragskosmetik, Verschleierungstaktik}

Wie zu erwarten war, wird jetzt von den Verfassungsgegnern hier und da gefordert, auch den neuen Vertrag abzulehnen, weil er außer ,Symbolik“ und „Präsentation“ an der Verfassung nichts ändere. Das ist genauso unbegründet wie die tröstende Behauptung der Verfassungsfreunde, die Substanz habe nicht gelitten, es seien ja „nur“ Symbolik und Präsentation geändert worden. Die Substanz des Verfassungsvertrages ist zwar gerettet worden - das ist es, was zählt - aber die Schrammen und Verletzungen, die die Verhandlungen im Europäischen Rat und das Mandat für die Regierungskonferenz am Verfassungsvertrag, und nicht nur an ihm, hinterlassen haben, sind tiefer als auf den ersten Blick zu erkennen ist. Zu ihnen zähle ich nicht die Versetzung der Grundrechte-Charta in ein Protokoll oder eine Erklärung, nicht die Großbritannien gewährten ,opt-outs', auch nicht das Hinausschieben der Anwendung der doppelten Mehrheit und den ,Ioannina-Mechanismus'.

Im neuen Text wird nicht mehr von der „Union der Bürger und der Staaten“ die Rede sein, sondern weiterhin nur von den „Hohen vertragschließenden Parteien“. Die Staaten also bilden die Union. Sie bleibt - Anerkennung der Rechtspersönlichkeit und Berufung auf die Rechtsprechung des Europäischen Gerichtshofs hinsichtlich des Vorrangs des Unionsrechts vor dem nationalen Hin oder Her - ein Staatenverbund. Nicht nur die vergemeinschafteten, also föderalen, sondern auch die intergouvernmentalen, also konföderalen, Elemente und Entscheidungsverfahren sind konstitutiv.

Die Mitgliedstaaten können der Europäischen Union übertragene Kompetenzen auch wieder entziehen. Das war zwar auch bisher schon so, und die explizite Aufnahme in den Vertrag trägt den bestehenden politischen Realitäten in Europa nur Rechnung, dennoch ist sie implizit deutlich mehr als nur die Erinnerung an eine Möglichkeit. Sie relativiert das alte Ziel, eine ,immer engere Union“ zu bilden. Das gleiche gilt übrigens auch für das politisch de facto immer vorhandene, nun aber wie schon in der Verfassung vertraglich garantierte Recht des Austritts aus der Union.

* Prof. Dr. Klaus Hänsch, MdEP, ehem. Präsident des Europäischen Parlaments und Präsidiumsmitglied des Verfassungskonvents. 
Die Wiedereinsetzung von „Verordnung“ und „Richtlinie“ anstelle der neuen Bezeichnungen „Europäisches Gesetz“ und „Europäisches Rahmengesetz“ sowie die Rücknahme des Titels „Europäischer Außenminister“ sind mehr als Vertragskosmetik zur Weichzeichnung des ,Verfassungs-Profils‘. Dahinter steckt schlicht politische Feigheit - eine durchaus symptomatische. Die niederländische Regierung vor allem wollte die politischen und rechtlichen Realitäten in der Union vor den Bürgerinnen und den Bürgern verschleiern. Wenn sich die Europapolitik schon weigert, die Dinge bei den Namen zu nennen, die die Bürger verstehen, verwundert es nicht, dass sie die Herausbildung emotionaler Bindungen an die Europäische Union auch durch den Verzicht auf die Nennung der Symbole wie Fahne und Hymne verhindern will.

Die Umwandlung des Verfassungsvertrages in einen Änderungsvertrag zu den bestehenden Verträgen war gewiss die einzige verbliebene Chance, die Substanz zu retten. Allerdings haben sich die Regierungen damit heimlich und kaum umkehrbar, von einem zentralen Teil des Mandats verabschiedet, das sie in Laeken dem Verfassungskonvent erteilt hatten: in den Grundlagen des Primärrechts der Union mehr Transparenz zu schaffen. Mehr noch: Die Notwendigkeit, die Reformen des Verfassungsvertrages in einem Änderungsvertrag bis zur Unauffindbarkeit zu verstecken, trägt der Tatsache Rechnung, dass für die Bevölkerung in der Mehrzahl der Mitgliedstaaten die Grenze der Integrationsbereitschaft erreicht ist. Volksabstimmungen über einen Verfassungsentwurf entfachen keinen neuen Europa-Enthusiasmus. Für einige der Verfassungsfreunde ist das eine ebenso bittere wie späte Erkenntnis.

\section{Künftige Entwicklung europäischer Einigung}

Gesetzt, es gelingt der Regierungskonferenz unter portugiesischem Vorsitz, das Mandat des Europäischen Rats wie vorgesehen bis zum Lissabon-Gipfel im Oktober in einen konkreten Änderungsvertrag zu überführen und, gesetzt, alle 27 Mitgliedstaaten ratifizieren ihn, zeichnet sich die folgende Entwicklung der Einigung Europas ab.

\section{Justierungen statt Vertragsreform}

In den Vertragsbeschlüssen von Maastricht, Amsterdam und Nizza wurde jeweils die nächste Reform mehr oder weniger explizit angekündigt. Kommt der Reformvertrag zustande, wird sich so schnell keiner der Mitgliedstaaten noch einmal auf das Abenteuer einer substanziellen Vertragsreform oder gar der Ausarbeitung einer Verfassung einlassen. Er wird, von kleineren Justierungen und Reparaturen abgesehen, über einen längeren Zeitraum hinweg nicht mehr verändert werden - jedenfalls solange die 27 in der Union zusammenbleiben.

\section{Selbstbehauptung Europas}

In den neuen Vertragsbestimmungen für das gemeinsame Handeln der Union nach außen findet der sich seit Längerem ankündigende Paradigmenwechsel der europäischen Integration seine vertragsrechtliche Entsprechung. Die Antriebskraft für die Einigung Europas verlagert sich von der Sicherung des Friedens und der Herstellung des Binnenmarktes in Europa auf die wirtschaftliche und politische Selbstbehauptung Europas in der Welt. Das entspricht dem in allen Meinungsumfragen zu erkennenden Verlangen einer großen Mehrheit der Bürger, dass Europa in der Welt ,mit einer Stimme“ sprechen soll. 


\section{Permanenter Begründungszwang}

Anders als die Nationalstaaten, die sich durch Staatsvolk, Staatsgebiet und Staatsgewalt aus sich selbst heraus begründen, unterliegt die Union weiterhin einem permanenten Begründungszwang. Die neuen Bestimmungen zur Klimapolitik und zur Energiesolidarität sowie die aus dem Verfassungsvertrag übernommenen Zielsetzungen in Art. 3 für die Rolle Europas in der Welt haben mehr als nur eine deklaratorische Bedeutung. Mit ihnen gibt der neue Vertrag eine zeitgemäß ergänzte Antwort auf die alte Frage: „Wozu einigen wir Europa?“

\section{Neues institutionelles Gleichgewicht}

Die nationalen Parlamente werden nicht nur weiter gestärkt, sie erhalten auch erstmals einen eigenen Artikel in den Verträgen. Die Volksvertretungen der nationalen Ebene werden nunmehr in die europäische Ebene mit erheblichen Mitberatungs- und Kontrollrechten einbezogen. Staats- und Verfassungsrechtlern muss das im vergleichenden Blick auf nationale Verfassungen als Systembruch erscheinen. Tatsächlich ist es eine Konsequenz aus der ,suigeneris-Struktur" der Union als Staatenverbund, in der die Volksvertretungen der Mitgliedstaaten zwar nicht an der Rechtsetzung auf Unionsebene unmittelbar, aber an der Einhaltung der Prinzipien von Subsidiarität und Verhältnismäßigkeit mitentscheiden müssen. Ob es dabei bleibt oder ob die Handlungsfähigkeit der Union durch die Übertragung innerpolitischer Konflikte auf die europäische Ebene leidet, wird die Praxis zeigen.

Der Reformvertrag stärkt die Institutionen und stellt zwischen ihnen ein neues Gleichgewicht her. Das hat ein erhebliches Entwicklungspotenzial. Wie weit es reicht, wird sich erst in der Vertragspraxis erweisen. Die Auswirkungen auf Sichtbarkeit, Dynamik und Legitimation der europäischen Politik könnten durchaus größer sein, als sich aus dem bloßen Text des Vertrages ergibt. Wie groß, wird in starkem Maße von den Personen abhängen, die diese Ämter als erste bekleiden. Welche Bedeutung und Kraft wächst dem neugeschaffenen Amt des gewählten Präsidenten des Europäischen Rates zu? Wird der sogenannte „Doppelhut“ auf dem Kopf des so nicht genannten Außenministers diesem die Beachtung und das Gewicht verleihen, das mit dieser hybriden Konstruktion angestrebt wird? Kann der Präsident der EU-Kommission ein Gleichgewicht herstellen zwischen seiner Rolle als Chef der Brüsseler Administration, die die Hüterin der Verträge ist, und als Chef einer Quasi-Regierung, die sich auf eine politische Mehrheit im Parlament stützen muss?

\section{Keine „Neugründung “ der Union}

Jeder Gedanke an eine „Neugründung“ der Union, wie er hier und da während des Ratifizierungsprozesses des Verfassungsvertrages propagiert wurde, ist vom Tisch. Intellektuelle Nebelkerzen bleiben auch EU-Fortentwicklungsmodelle wie ,Kerneuropa', ,konzentrische Kreise', ,zwei (oder mehrere) Geschwindigkeiten“ und so weiter. Das Instrument der ,Verstärkten Zusammenarbeit" innerhalb des Vertrages hat sich bisher schon als unwirksam erwiesen. Das wird sich nicht grundlegend ändern. $\mathrm{Zu}$ beachten ist allerdings, dass es ironischerweise automatisch zu einer ,Verstärkten Zusammenarbeit ' der übrigen Mitgliedstaaten kommt, wenn Großbritannien von seinen neugewährten , opt outs ' Gebrauch macht. Außerhalb des Vertrages können sich selbstverständlich weiterhin Pioniergruppen nach dem Beispiel ,Schengen“ bilden. Allerdings bleibt für ein solches Vorgehen nur noch wenig Raum, am ehesten noch im Bereich polizeiliche Zusammenarbeit, wie das Abkommen von Prüm zeigt, sowie bei Militär und Rüstung und einzelnen industriellen Großprojekten. Grundsätzlich bleibt die Gefahr bestehen, dass aus einer, Verstärkten Zusammenarbeit` weniger ein ,geschwächter Zusammenhalt` aller wird und die Union zu einer Patchwork-Union degeneriert. 


\section{Neues Machtgefüge}

Der ungewöhnlich erfolgreiche Gipfel vom Juni 2007 lässt ein neues Machtgefüge zwischen den EU-Mitgliedstaaten erkennen. Deutschlands Rolle als Orientierungsmacht in der Union ist wieder gestärkt. Es kann zwar nicht alles durchsetzen, aber ohne oder gar gegen Deutschland wird zumindest in Grundsatzfragen kaum etwas zustande kommen.

Erfreulicherweise ist Frankreich wieder da - aber wo steht es? Es mag sein, dass Sarkozys Positionen zum Beitritt der Türkei, zur Währungspolitik, zu Wirtschaftsregierung und Wettbewerb und so weiter die Europäische Union stärker als bisher auf Nützen und Schützen orientieren sollen - auf Aufgaben also, wie sie jede staatliche Organisation erfüllen muss. Aber noch ist nicht zu erkennen, ob sich die irrlichternde Dynamik zu der Kraft entwickelt, die in der Ruhe liegt.

Großbritannien bleibt bei der bisherigen Grundorientierung der britischen Europapolitik: „Dabei sein, um zu verhindern“. Es gerät überdies durch die ,opt-outs“ noch stärker in die Gefahr, randständig zu werden - es sei denn, der Zauber, der jedem Anfang innewohnt, hilft dem Premierminister, Commons und Lords davon zu überzeugen, dass das Vereinigte Königreich den Reformvertrag nicht als letztes, sondern als erstes Land in der Europäischen Union ratifizieren sollte.

Polen hat sich gegenwärtig ,am weitesten vom europäischen Geist entfernt“ (Romano Prodi) - nicht durch das Beharren auf der Quadratwurzel für die Stimmengewichtung im Rat, sondern durch die ausschließlich und polemisch gegen ein anderes EU-Mitgliedsland gerichteten Begründungen dafür. Meinungsumfragen signalisieren zwar, dass die Mehrheit der Polen sehr wohl in der Europäischen Union angekommen zu sein scheint, aber für die polnische Regierung gilt das gewiss nicht. Sie hat das hohe Ansehen Polens in kurzer Zeit fast auf Null reduziert und übersehen, dass in der Union das Ansehen eines Staates und seiner Regierung ein höherer Machtfaktor sein kann als Größe und Zahl. Einer, wie Luxemburgs Premierminister Jean-Claude Juncker, beweist es immer wieder aufs Neue.

\section{Ratifizierungsprobleme}

Die Aussicht, dass der Ratifizierungsprozess in allen Mitgliedstaaten erfolgreich zu Ende gebracht werden kann, ist keineswegs besser als vor dem französischen und niederländischen „Nein“ zum Verfassungsvertrag. Die Staaten, die den Verfassungsvertrag ratifiziert hatten, werden wohl auch dem neuen Vertrag ihre Zustimmung geben. Frankreich wird keine Schwierigkeiten mehr machen. Die Aussichten in Dänemark, Schweden und Portugal sind durchaus gut. Aber in Polen ist die politische Lage völlig unübersichtlich geworden. In Tschechien halten sich weiterhin Befürworter und Ablehner, sowohl im Parlament als auch in der Bevölkerung, die Waage. In den Niederlanden könnte es erneut zu einem Referendum kommen mit der Gefahr eines zweiten „Nee“. Dass Gordon Brown seine Strategie, auf eine Volksabstimmung zu verzichten und den Vertrag allein im Parlament ratifizieren zu lassen, durchhalten kann, wird mit jedem Tag, an dem in seiner eigenen Fraktion die Zahl der Befürworter eines Referendums - und damit der potenziellen Gegenstimmen im Parlament wächst, immer unwahrscheinlicher. Vor diesem Hintergrund sollte jeder Volksentscheid über den Verfassungsvertrag zu einer Abstimmung über den Verbleib des Landes in der Union gemacht werden. 